\title{
A generalization and an application of the arithmetic-geometric mean inequality for the Frobenius norm
}

\section{Xuesha $\mathrm{Wu}^{1 *}$}

\section{"Correspondence:}

xuesha_wu@163.com

'School of Marxism and General Education, Chongqing College of Electronic Engineering, Chongqing, P.R. China

\begin{abstract}
Recently, Kittaneh and Manasrah (J. Math. Anal. Appl. 361:262-269, 2010) showed a refinement of the arithmetic-geometric mean inequality for the Frobenius norm. In this paper, we shall present a generalization of Kittaneh and Manasrah's result. Meanwhile, we will also give an application of Kittaneh and Manasrah's result. That is, we obtain an improvement of Jocić and Kittaneh's inequality which was presented in (Jocić and Kittaneh in J. Oper. Theory 31:3-10, 1994).
\end{abstract}

MSC: $15 \mathrm{~A} 42 ; 47 \mathrm{~A} 63$

Keywords: Unitarily invariant norms; Arithmetic-geometric mean inequality; Positive semidefinite matrices

\section{Introduction}

Let $M_{n}(C)$ be the space of $n \times n$ complex matrices. Let $\|\cdot\|$ denote any unitarily invariant norm on $M_{n}(C)$. For $A \in M_{n}(C)$, the Frobenius norm of $A$ is defined by $\|A\|_{F}=\sqrt{\operatorname{tr}\left(A^{*} A\right)}$, where $\operatorname{tr}(X)$ is the trace of $X$. It is known that the Frobenius norm is unitarily invariant.

Let $A, B \in M_{n}(C)$ be positive semidefinite. Bhatia and Kittaneh proved in [1] that

$$
\left\|A^{1 / 2} B^{1 / 2}\right\| \leq\left\|\frac{A+B}{2}\right\|
$$

which is known as the arithmetic-geometric mean inequality for unitarily invariant norms.

Let $A, X, B \in M_{n}(C)$ and suppose that $A$ and $B$ are positive semidefinite. Bhatia and Davis proved in [2] that

$$
\left\|A^{1 / 2} X B^{1 / 2}\right\| \leq\left\|\frac{A X+X B}{2}\right\|
$$

which is a generalization of inequality (1.1).

(c) The Author(s) 2018. This article is distributed under the terms of the Creative Commons Attribution 4.0 International License (http://creativecommons.org/licenses/by/4.0/), which permits unrestricted use, distribution, and reproduction in any medium, provided you give appropriate credit to the original author(s) and the source, provide a link to the Creative Commons license, and indicate if changes were made. 
Recently, Kittaneh and Manasrah [3] showed a refinement of inequality (1.2) for the Frobenius norm, which can be stated as follows:

$$
\left\|A^{1 / 2} X B^{1 / 2}\right\|_{F}+\frac{1}{2}\left(\sqrt{\|A X\|_{F}}-\sqrt{\|X B\|_{F}}\right)^{2} \leq\left\|\frac{A X+X B}{2}\right\|_{F} .
$$

The authors of [4] and [5] gave some generalizations of inequality (1.3).

Let $A, X, B \in M_{n}(C)$ such that $A$ and $B$ are self-adjoint. Jocić and Kittaneh proved in [6] that for $n \in N$ and $j=1, \ldots, n$,

$$
\left\|A^{n+j} X B^{n-j+1}-A^{n-j+1} X B^{n+j}\right\| \leq\left\|A^{n+j+1} X B^{n-j}-A^{n-j} X B^{n+j+1}\right\| .
$$

Bhatia gave a simple proof of inequality (1.4) in [7]. For more information on inequalities of unitarily invariant norms, the reader is referred to [8-12] and the references therein.

In this short note, we first present a new generalization of inequality (1.3). After that, as an application of inequality (1.3), we show a refinement of inequality (1.4) for the Frobenius norm.

\section{Main results}

In this section, we show the main results of this paper. To do this, we need the following lemmas.

Lemma 2.1 ([11]) Let $A, X, B \in M_{n}(C)$. If $\alpha \in[0,1]$, then

$$
\left\|A^{*} X B\right\|^{2} \leq\left\|\alpha A A^{*} X+(1-\alpha) X B B^{*}\right\|\left\|(1-\alpha) A A^{*} X+\alpha X B B^{*}\right\| .
$$

Lemma 2.2 Let $A, X, B \in M_{n}(C)$. Then

$$
\left\|A^{*} X B\right\|_{F}+\frac{1}{2}\left(\sqrt{\left\|A A^{*} X\right\|_{F}}-\sqrt{\left\|X B B^{*}\right\|_{F}}\right)^{2} \leq\left\|\frac{A A^{*} X+X B B^{*}}{2}\right\|_{F} .
$$

Proof By the polar decomposition of matrices and the properties of unitary invariant norms, we know that inequality (1.3) is equivalent to Lemma 2.2. This completes the proof.

Theorem 2.1 Let $A, X, B \in M_{n}(C), \alpha \in[0,1]$ such that $A, B$ are positive semidefinite. Then

$$
\left\|A^{1 / 2} X B^{1 / 2}\right\|_{F}+\frac{1}{2}(\sqrt{C(\alpha)}-\sqrt{C(1-\alpha)})^{2} \leq\left\|\frac{A X+X B}{2}\right\|_{F}
$$

where

$$
C(\alpha)=\|\alpha A X+(1-\alpha) X B\|_{F}, \quad C(1-\alpha)=\|(1-\alpha) A X+\alpha X B\|_{F} .
$$

Proof By definition of the Frobenius norm, we have

$$
\begin{aligned}
C^{2}(\alpha) & =\|\alpha A X+(1-\alpha) X B\|_{F}^{2} \\
& =\alpha^{2}\|A X\|_{F}^{2}+(1-\alpha)^{2}\|X B\|_{F}^{2}+2 \alpha(1-\alpha)\left\|A^{1 / 2} X B^{1 / 2}\right\|_{F}^{2},
\end{aligned}
$$




$$
\begin{aligned}
C^{2}(1-\alpha) & =\|(1-\alpha) A X+\alpha X B\|_{F}^{2} \\
& =(1-\alpha)^{2}\|A X\|_{F}^{2}+\alpha^{2}\|X B\|_{F}^{2}+2 \alpha(1-\alpha)\left\|A^{1 / 2} X B^{1 / 2}\right\|_{F}^{2},
\end{aligned}
$$

and so

$$
\|A X\|_{F}^{2}+\|X B\|_{F}^{2}-C^{2}(\alpha)-C^{2}(1-\alpha)=2 \alpha(1-\alpha)\|A X-X B\|_{F}^{2} .
$$

It follows from (2.1) and (2.3) that

$$
\begin{aligned}
\| A X+ & X B \|_{F}^{2}-\left((\sqrt{C(\alpha)}-\sqrt{C(1-\alpha)})^{2}+2\left\|A^{1 / 2} X B^{1 / 2}\right\|_{F}\right)^{2} \\
& -2 \alpha(1-\alpha)\|A X-X B\|_{F}^{2} \\
= & \|A X\|_{F}^{2}+\|X B\|_{F}^{2}+2\left\|A^{1 / 2} X B^{1 / 2}\right\|_{F}^{2}-4\left\|A^{1 / 2} X B^{1 / 2}\right\|_{F}^{2} \\
& -2 \alpha(1-\alpha)\|A X-X B\|_{F}^{2}-(\sqrt{C(\alpha)}-\sqrt{C(1-\alpha)})^{4} \\
& -4\left\|A^{1 / 2} X B^{1 / 2}\right\|_{F}(\sqrt{C(\alpha)}-\sqrt{C(1-\alpha)})^{2} \\
= & \|A X\|_{F}^{2}+\|X B\|_{F}^{2}-2\left\|A^{1 / 2} X B^{1 / 2}\right\|_{F}^{2} \\
& -2 \alpha(1-\alpha)\|A X-X B\|_{F}^{2}-(\sqrt{C(\alpha)}-\sqrt{C(1-\alpha)})^{4} \\
& -4\left\|A^{1 / 2} X B^{1 / 2}\right\|_{F}(\sqrt{C(\alpha)}-\sqrt{C(1-\alpha)})^{2} \\
\geq & \|A X\|_{F}^{2}+\|X B\|_{F}^{2}-2 C(\alpha) C(1-\alpha)-(\sqrt{C(\alpha)}-\sqrt{C(1-\alpha)})^{4} \\
& -4\left\|A^{1 / 2} X B^{1 / 2}\right\|_{F}(\sqrt{C(\alpha)}-\sqrt{C(1-\alpha)})^{2}-2 \alpha(1-\alpha)\|A X-X B\|_{F}^{2} \\
= & C^{2}(\alpha)+C^{2}(1-\alpha)-2 C(\alpha) C(1-\alpha)-(\sqrt{C(\alpha)}-\sqrt{C(1-\alpha)})^{4} \\
& -4\left\|A^{1 / 2} X B^{1 / 2}\right\|_{F}(\sqrt{C(\alpha)}-\sqrt{C(1-\alpha)})^{2} \\
= & (C(\alpha)-C(1-\alpha))^{2}-(\sqrt{C(\alpha)}-\sqrt{C(1-\alpha)})^{4} \\
& -4\left\|A^{1 / 2} X B^{1 / 2}\right\|_{F}(\sqrt{C(\alpha)}-\sqrt{C(1-\alpha)})^{2} \\
= & (\sqrt{C(\alpha)}-\sqrt{C(1-\alpha)})^{2}(\sqrt{C(\alpha)}+\sqrt{C(1-\alpha)})^{2} \\
& -(\sqrt{C(\alpha)}-\sqrt{C(1-\alpha)})^{4}-2\left\|A^{1 / 2} X B^{1 / 2}\right\|_{F}(\sqrt{C(\alpha)}-\sqrt{C(1-\alpha)})^{2} \\
= & 4(\sqrt{C(\alpha)}-\sqrt{C(1-\alpha)})^{2}\left(\sqrt{C(\alpha) C(1-\alpha)}-\left\|A^{1 / 2} X B^{1 / 2}\right\|_{F}\right) \\
\geq & 0 .
\end{aligned}
$$

That is,

$$
\begin{aligned}
& \left(2\left\|A^{1 / 2} X B^{1 / 2}\right\|_{F}+(\sqrt{C(\alpha)}-\sqrt{C(1-\alpha)})^{2}\right)^{2}+2 \alpha(1-\alpha)\|A X-X B\|_{F}^{2} \\
& \quad \leq\|A X+X B\|_{F}^{2},
\end{aligned}
$$

which implies inequality (2.2). This completes the proof.

Remark 2.1 Putting $\alpha=0$ or $\alpha=1$ in inequality (2.2), we can obtain inequality (1.3). So, inequality (2.2) is a generalization of inequality (1.3).

Next, we will show a refinement of inequality (1.4). 
Theorem 2.2 Let $A, X, B \in M_{n}(C)$ such that $A$ and $B$ are self-adjoint. If $n \in N$ and $j=$ $1, \ldots, n$, then we have

$$
\left\|A^{n+j} X B^{n-j+1}-A^{n-j+1} X B^{n+j}\right\|_{F}+K^{2}(n, j) \leq\left\|A^{n+j+1} X B^{n-j}-A^{n-j} X B^{n+j+1}\right\|_{F},
$$

where

$$
\begin{aligned}
K(n, j)= & \sqrt{\left\|A^{2}\left(A^{n+j-1} X B^{n-j}-A^{n-j} X B^{n+j-1}\right)\right\|_{F}} \\
& -\sqrt{\left\|\left(A^{n+j-1} X B^{n-j}-A^{n-j} X B^{n+j-1}\right) B^{2}\right\|_{F}} .
\end{aligned}
$$

Proof We prove it by induction. For $j=1$ and any positive integer $n$, by Lemma 2.2 and the triangle inequality for unitary invariant norms, we have

$$
\begin{aligned}
& \left\|A^{n+1} X B^{n}-A^{n} X B^{n+1}\right\|_{F}+\frac{1}{2} K^{2}(n, 1) \\
& \quad=\left\|A\left(A^{n} X B^{n-1}-A^{n-1} X B^{n}\right) B\right\|_{F}+\frac{1}{2} K^{2}(n, 1) \\
& \quad \leq \frac{1}{2}\left\|A^{2}\left(A^{n} X B^{n-1}-A^{n-1} X B^{n}\right)+\left(A^{n} X B^{n-1}-A^{n-1} X B^{n}\right) B^{2}\right\|_{F} \\
& \quad=\frac{1}{2}\left\|A^{n+2} X B^{n-1}-A^{n-1} X B^{n+2}+A^{n} X B^{n+1}-A^{n+1} X B^{n}\right\|_{F} \\
& \quad \leq \frac{1}{2}\left\|A^{n+2} X B^{n-1}-A^{n-1} X B^{n+2}\right\|_{F}+\frac{1}{2}\left\|A^{n+1} X B^{n}-A^{n} X B^{n+1}\right\|_{F}
\end{aligned}
$$

which is equivalent to

$$
\left\|A^{n+1} X B^{n}-A^{n} X B^{n+1}\right\|_{F}+K^{2}(n, 1) \leq\left\|A^{n+2} X B^{n-1}-A^{n-1} X B^{n+2}\right\|_{F} .
$$

Now, suppose that Theorem 2.2 has been proved for $j-1$. By Lemma 2.2, the triangle inequality for unitary invariant norms, and induction hypothesis, we have

$$
\begin{aligned}
& \left\|A^{n+j} X B^{n-j+1}-A^{n-j+1} X B^{n+j}\right\|_{F}+\frac{1}{2} K^{2}(n, j) \\
& \quad=\left\|A\left(A^{n+j-1} X B^{n-j}-A^{n-j} X B^{n+j-1}\right) B\right\|_{F}+\frac{1}{2} K^{2}(n, j) \\
& \quad \leq \frac{1}{2}\left\|A^{2}\left(A^{n+j-1} X B^{n-j}-A^{n-j} X B^{n+j-1}\right)+\left(A^{n+j-1} X B^{n-j}-A^{n-j} X B^{n+j-1}\right) B^{2}\right\|_{F} \\
& \quad=\frac{1}{2}\left\|A^{n+j+1} X B^{n-j}-A^{n-j} X B^{n+j+1}+A^{n+j-1} X B^{n-(j-1)+1}-A^{n-(j-1)+1} X B^{n+j-1}\right\|_{F} \\
& \quad \leq \frac{1}{2}\left\|A^{n+j+1} X B^{n-j}-A^{n-j} X B^{n+j+1}\right\|_{F}+\frac{1}{2}\left\|A^{n+j-1} X B^{n-(j-1)+1}-A^{n-(j-1)+1} X B^{n+j-1}\right\|_{F} \\
& \quad \leq \frac{1}{2}\left\|A^{n+j+1} X B^{n-j}-A^{n-j} X B^{n+j+1}\right\|_{F}+\frac{1}{2}\left\|A^{n+j} X B^{n-j+1}-A^{n-j+1} X B^{n+j}\right\|_{F},
\end{aligned}
$$

which is equivalent to

$$
\left\|A^{n+j} X B^{n-j+1}-A^{n-j+1} X B^{n+j}\right\|_{F}+K^{2}(n, j) \leq\left\|A^{n+j+1} X B^{n-j}-A^{n-j} X B^{n+j+1}\right\|_{F} .
$$

This completes the proof. 


\section{Acknowledgements}

The author wishes to express her heartfelt thanks to the referees for their detailed and helpful suggestions for revising the manuscript.

Funding

Not applicable.

\section{Competing interests}

The author declares that there is no conflict of interests regarding the publication of this paper.

\section{Authors' contributions}

The author read and approved the final manuscript.

\section{Publisher's Note}

Springer Nature remains neutral with regard to jurisdictional claims in published maps and institutional affiliations.

Received: 12 May 2018 Accepted: 11 June 2018 Published online: 20 June 2018

\section{References}

1. Bhatia, R., Kittaneh, F.: On the singular values of a product of operators. SIAM J. Matrix Anal. Appl. 11, 272-277 (1990)

2. Bhatia, R., Davis, C.: More matrix forms of the arithmetic-geometric mean inequality. SIAM J. Matrix Anal. Appl. 14, 132-136 (1993)

3. Kittaneh, F., Manasrah, Y.: Improved Young and Heinz inequalities for matrices. J. Math. Anal. Appl. 361, 262-269 (2010)

4. Wang, S., Zou, L., Jiang, Y.: Some inequalities for unitarily invariant norms of matrices. J. Inequal. Appl. 2011,10 (2011)

5. He, C., Zou, L., Qaisar, S.: On improved arithmetic-geometric mean and Heinz inequalities for matrices. J. Math. Inequal. 6, 453-459 (2012)

6. Jocić, D., Kittaneh, F.: Some perturbation inequalities for self-adjoint operators. J. Oper. Theory 31, 3-10 (1994)

7. Bhatia, R.: A simple proof of an operator inequality of Jocić and Kittaneh. J. Oper. Theory 31, 21-22 (1994)

8. Hu, X., Xue, J.: A note on reverses of Young type inequalities. J. Inequal. Appl. 2015, 98 (2015)

9. Hu, X: Young type inequalities for matrices. J. East China Norm. Univ. Natur. Sci. Ed. 4, 12-17 (2012)

10. Hu, X.: Some inequalities for unitarily invariant norms. J. Math. Inequal. 6, 615-623 (2012)

11. Zou, L., Jiang, Y.: A note on interpolation between the arithmetic-geometric mean and Cauchy-Schwarz matrix norm inequalities. J. Math. Inequal. 10, 1119-1122 (2016)

12. Zou, L.: An arithmetic-geometric mean inequality for singular values and its applications. Linear Algebra Appl. 528 25-32 (2017)

\section{Submit your manuscript to a SpringerOpen ${ }^{\circ}$ journal and benefit from:}

- Convenient online submission

- Rigorous peer review

Open access: articles freely available online

- High visibility within the field

Retaining the copyright to your article 